\title{
Probability Control for Verification of an Event Report Using Fuzzy System
}

\author{
Hyun Woo Lee, Soo Young Moon, Tae Ho Cho \\ School of Information and Communication Engineering, Sungkyunkwan University, Suwon, Republic of Korea \\ E-mail: \{hwoolee, moonmous, taecho\}@ece.skku.ac.kr \\ Received September 5, 2011; revised October 11, 2011; accepted November 182011
}

\begin{abstract}
Sensor networks include numerous sensor nodes that are vulnerable to physical attacks from the outside because they operate in open environments. The sensor nodes are compromised by an attacker. The compromised nodes generate false reports and inject the reports into sensor networks. The false report injection attacks deplete energy of the sensor nodes. Ye et al. proposed Statistical En-Route Filtering (SEF) to defend sensor nodes against the false report injection attacks. In SEF, sensor nodes verify the event reports based on a fixed probability. Thus, the verification energy of a node is the same whether the report is false or valid. But when there are few false reports, energy for verifying legitimate reports may be wasted. In this paper, we propose a method in which each node controls a probability of attempts at verification of an event report to reduce the wasted energy. The probability is determined through consideration of the number of neighboring nodes, the number of hops from the node to the sink node, and the rate of false reports among the 10 most recent event reports forwarded to a node. We simulated our proposed method to prove its energy efficiency. After the simulation, we confirmed that the proposed method is more efficient than SEF for saving sensor node's energy.
\end{abstract}

Keywords: Sensor Network, Statistical En-Route Filtering, False Report Injection Attack

\section{Introduction}

Recent Developments in micro-electro-mechanical systems (MEMS) technology and advances in wireless communications have enabled the growth of sensor networks [1]. Figure 1 shows a sensor network.

The sensor network is composed of many tiny sensor

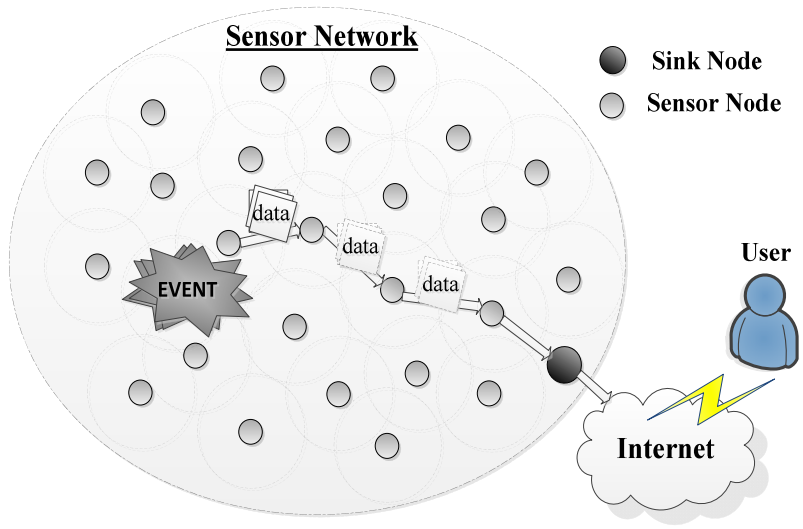

Figure 1. Sensor network. nodes each of which has limited computational, commu nicational, and sensing capabilities [1]. The sensor nodes coordinate to perform a common task [1]. Sensor networks are employed for a wide variety of applications, including industrial, military, biomedical, and environmental areas. Sensor network nodes are deployed in open environments in many applications [1,2]. Hence, the sensor nodes are vulnerable to physical attacks which compromise their cryptographic keys [1]. One such attack is false report injection attack. If an attacker compromises any node to obtain the security information, the attacker makes a compromised node generate false reports and insert them into the sensor network. A false report injection attack can result in not only a reduction of the already limited energy of sensor nodes in a battery powered network but also false alarms [3-6]. To minimize such damages, false reports have to be dropped en-route as soon as possible, while few eluded false reports have to be rejected at the sink node [4]. Fan Ye et al. proposed a solution that drops the false reports en-route called statistical en-route filtering (SEF). In SEF, each intermediate node includes authentication keys that 
verify reports from different partitions in a global pool [5]. Whenever a report is forwarded, each node verifies whether a report is legitimate. Legitimate report, the reports are forwarded to the next intermediate node. Nonlegitimate reports are dropped. Thus, the false reports that are generated by compromised nodes are filtered early, meaning that sensor nodes do not need to waste energy forwarding many false reports. However, when there are few false reports, the sensor nodes have to waste energy verifying both legitimate and false reports with the same probability [5].

In this paper, to save the energy that is consumed verifying event reports, we propose a method that controls a probability of attempts at verification of an event report through a fuzzy system in a sensor network. The prob- ability is decided by three elements: the number of nei- ghbor nodes, the number of hops from a node to a sink node and the rate of false reports.

Our proposed method is described in detail as follows. Section 2 explains SEF related work. Section 3 describes the proposed method. Section 4 shows the simulation results. Section 5 presents the study conclusion.

\section{Statistical En-Route Filtering}

SEF is composed of four steps: key assignment, report generation, en-route filtering, and sink verification. In this section, these four steps are explained

\subsection{Key Assignment}

Some of the keys in the global key pool are assigned to each sensor node. The keys are selected at random before the sensor nodes are deployed in the sensor field. The global key pool is divided into several non-overlapping partitions that have the same number of keys. Several partitions are randomly selected from the global key pool by a user, who then assigns some of the keys to a node. The number of keys assigned to each node is decided by the user. Each node generates Message authentication codes (MAC) using its keys to verify reports.

\subsection{Report Generation}

After key assignment and node deployment, when an event is occurred in the sensor field, multiple nodes that detect the event elect a center of stimulus node $(\mathrm{CoS})$, which most strongly detects the event. Each node that detects the event randomly chooses a key among its own keys that is used to generate a MAC. The MAC and the key index are sent to the CoS node, which then generates an event report to which the MACs received from the multiple nodes are attached. The report including the
MACs is forwarded to next intermediate node toward the sink node.

\subsection{En-Route Filtering}

Because of the random key assignment, each intermediate node has a probability that an intermediate node has a key that can verify a report. When a report is arrived at a node, the node uses one of its own keys to generate a MAC. Each node compares the number of key indices and MACs between the report and the node. If the node has a larger or smaller number of key indices and MACs than were decided by the user, or if key indices are derived from the same partitions, the report is dropped by the node. If neither situation occurs, the node finds a key that matches the one it chose. When there is a matching key with the key of the node, the node generates the MAC using the key. When a key matches that chosen by the node, the node generates a MAC, which is compared to the MAC of a report. If the MAC of the node matches the MAC of a report, the report is forwarded to next node. If the MACs do not match, the report is considered false and is dropped

\subsection{Sink Verification}

After en-route filtering, a few false reports can still arrive at the sink node because the intermediate nodes use the same probability to verify reports. However, in SEF, the sink node has of all keys that are in the global key pool. Thus, the sink node can filter the false reports. As stated above, in SEF, false reports can be dropped early and energy consumption in the sensor network can be reduced through use of en-route filtering.

\section{Proposed Method}

\subsection{Motivation}

In SEF, each node uses the same probability to verify a report regardless of its status (false vs. legitimate). Thus, if there are few or no false reports in the sensor network, the energy that is consumed verifying legitimate nodes is wasted. To save verification energy for legitimate reports, we propose a method that controls a probability of attempts at verification of an event report. Section 3.2 shows the assumption of our proposed method.

\subsection{Assumption}

The proposed method includes the following assumptions:

- Each node has a unique identification (ID). 
- Each node has a table which is composed of two values: IDs of neighbor nodes and a probability of attempts at verification of an event report of neighbor nodes.

- Each node stores the number of hops from the node to the sink node.

- Each node stores verification results of the ten most recent reports of every node.

\subsection{Operation}

Key assignment and node deployment occur the same way in the proposed method as in SEF. However, in the proposed method, after these steps, a unique ID is assigned to each node and a table is generated that consists of IDs of neighbor nodes and the probabilities that attempt to verify a report. Figure 2 shows a table that is composed of two elements: neighbor node IDs and attempts to verify event report probabilities.

As shown in Figure 2, the probabilities of neighbor nodes are equal to 1 , because the information for controlling the probability is not yet generated. After ID distribution for sensor nodes and table generation, the nodes are deployed and keys are assigned to the nodes. Reports are generated here the same way as in SEF. When an event occurs, the intermediate nodes that detect the event elect a $\mathrm{CoS}$ node to which MACs are forwarded. The $\mathrm{CoS}$ node generates the event report to which MACs collected from the intermediate nodes are attached. The event report is then forwarded to the next node toward the sink node. Every time the event report is forwarded to an intermediate node, that node verifies the event report. This en-route filtering step is the differenttiating feature of the proposed method from the SEF. Figures 3, 4 and 5 show a sample event report verification of the proposed method.

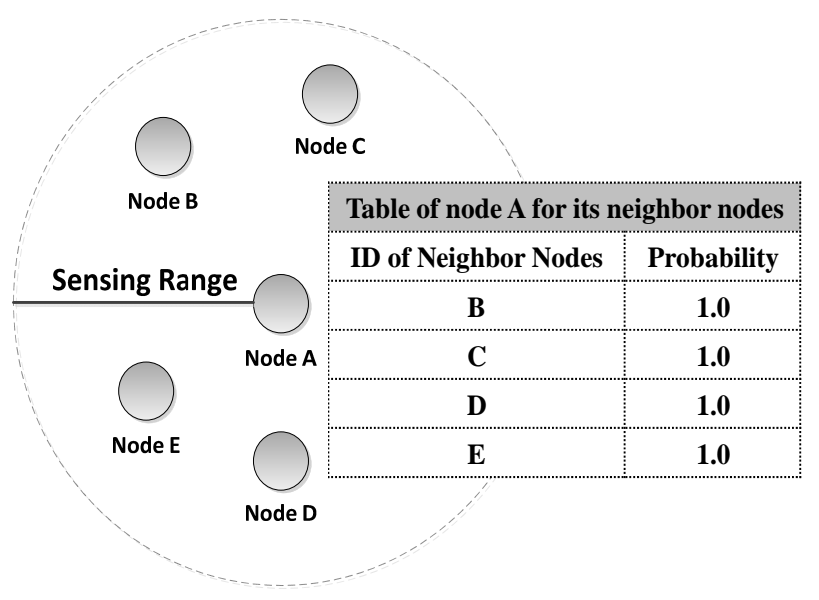

Figure 2. Event report verification information.

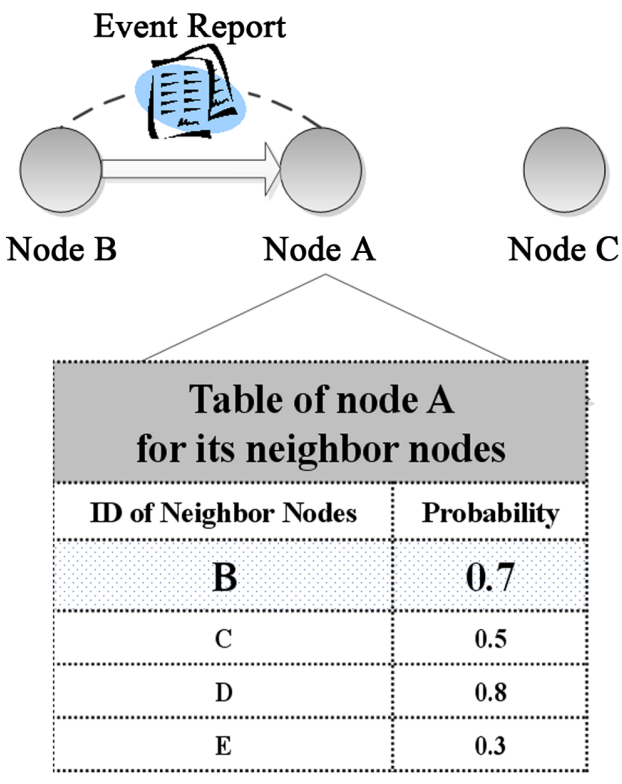

Figure 3. Check for the probability of the neighbor node.

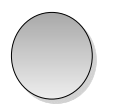

Node A

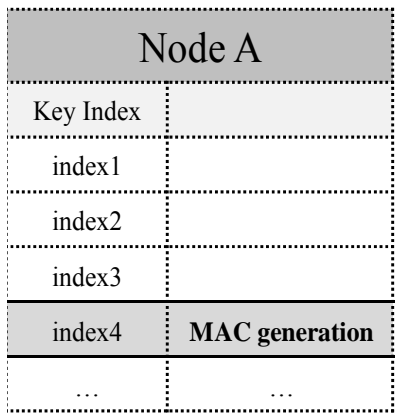

Figure 4. Verification of an event report.

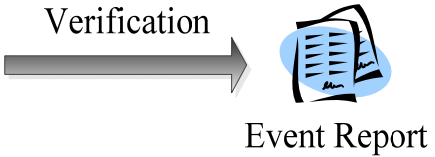

\begin{tabular}{|c:c|}
\hline Event Report \\
\hdashline Key Index & MAC \\
\hdashline index1 & MAC5 \\
\hline index4 & MAC4 \\
\hdashline index6 & MAC8 \\
\hdashline index7 & MAC9 \\
\hdashline$\ldots$ & $\ldots$ \\
\hdashline$\ldots$ & $\ldots$ \\
\hdashline
\end{tabular}

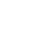

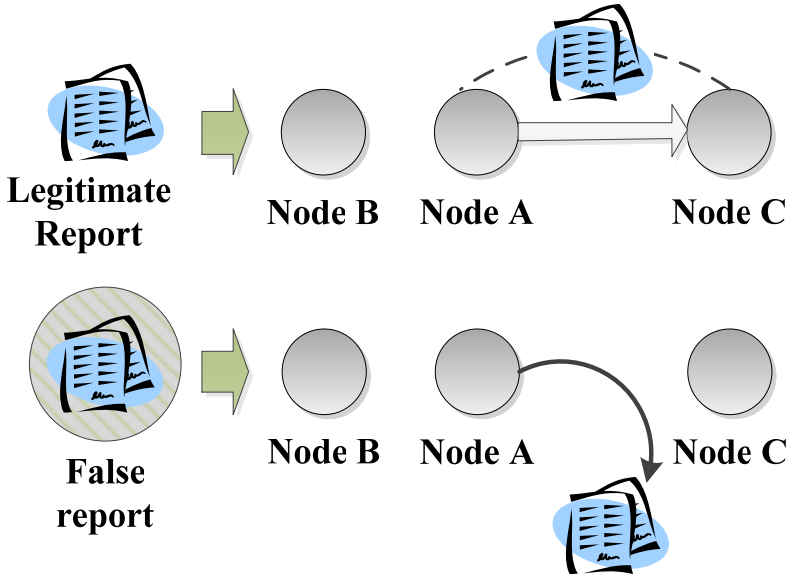

Figure 5. Results of verification for an event report. 
As shown Figure 3, when the each node receives the event report, the node checks identification of the neighbor node which sends to the node and attempt to verify a report probability. The node attempts to verify the report by the probability in Figure 4. After verification of the report, if the report is a legitimate report, the probability of the neighbor node becomes low. The report is forwarded to next node. But if the report is a false report, the probability of the neighbor node becomes high. The report is dropped in Figure 5. When an intermediate node receive an event report, if the probability of the neighbor node which forwards the event report to the intermediate node is high, lots of energy for verifying the report are consumed. On the contrary, if the probability is low, less energy is consumed than the probability. The probability is calculated by three inputs. Figure 6 shows three inputs and output that is probability of attempts at verification of a report using a fuzzy system.

The following figures are shown the three inputs which are used to calculate the probability

Figure 7 Shows a fuzzy membership function of the number of neighbor nodes. Figure 8 shows a fuzzy membership function of the number of from a node to a sink node. Figure 9, the fuzzy values of three fuzzy membership functions are in the range of $0-1$. The values belong to the fuzzy set, which is composed of three levels: small, medium, and large. A fuzzy membership function of a probability of attempts at verification of an event report comes from the three membership functions. Figure 10 shows an output fuzzy membership function of the probability.

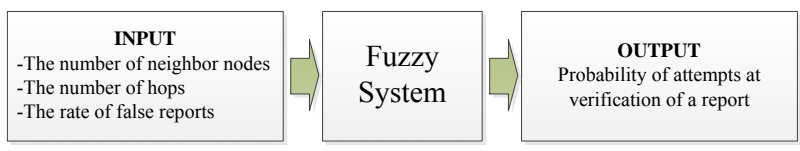

Figure 6. Input and output in fuzzy system.

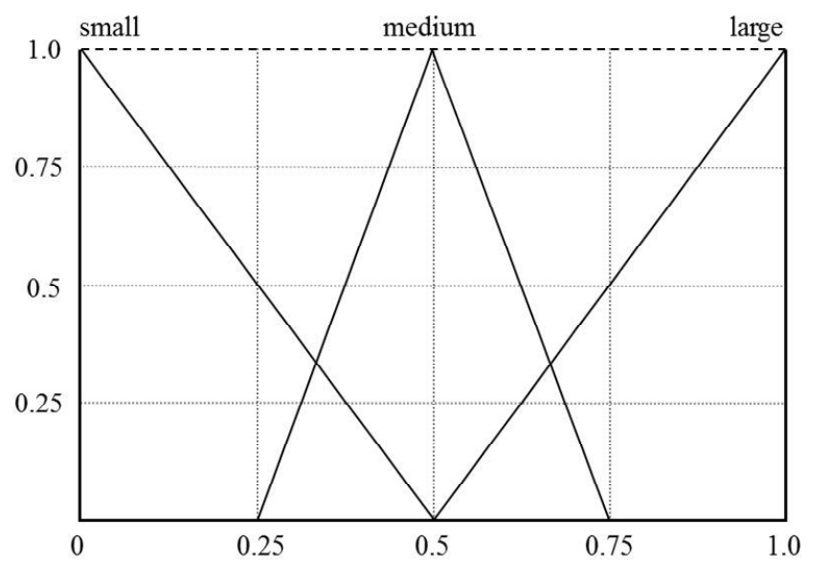

Figure 7. Fuzzy membership function for the number of neighbor nodes.

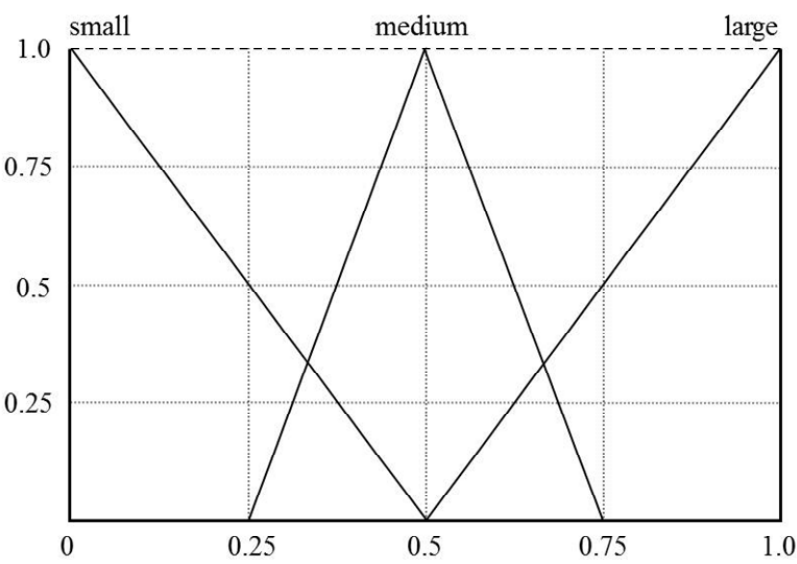

Figure 8. Fuzzy membership function for the number of hops from a node to sink node.

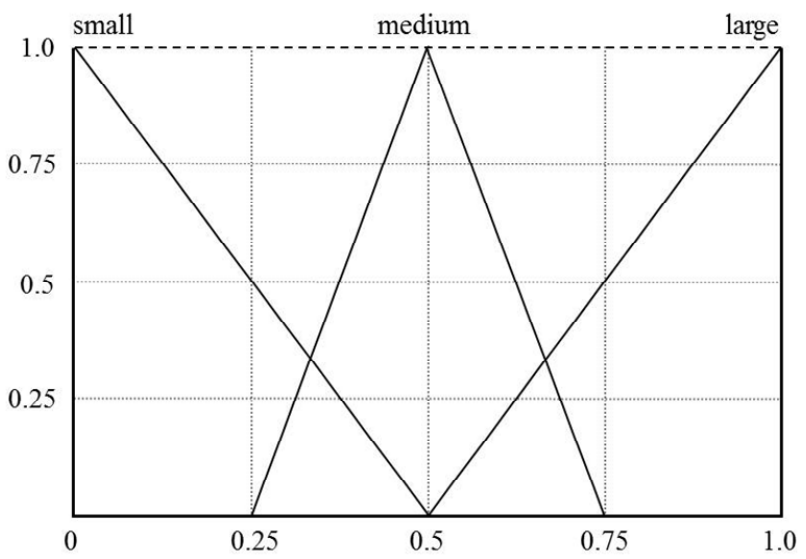

Figure 9. Fuzzy membership function for the rate of false reports.

Figure 10, a fuzzy value of the probability is in the range of $0-1$. The value belongs to the fuzzy set which is composed of five levels: very small, small, medium, large, and very large. The fuzzy membership functions are defined by fuzzy rules that are designed by a user.

The part of fuzzy rules of proposed method is shown in Table 1.

In our proposed method, each sensor node verifies a report controlling the probability of attempts at verification of an event report that is calculated by the fuzzy system for its neighbor nodes. The proposed method controls the probability and consumes less node's energy than SEF. A comparison of the energy efficiency between the proposed method and SEF is able to express some equations. Equation (1) represents the probability that a node includes a key that has not been compromised by an attacker [4]. Table 2 explains elements determining $P_{1}$

$$
P_{1}=\frac{k\left(T-N_{c}\right)}{N}
$$




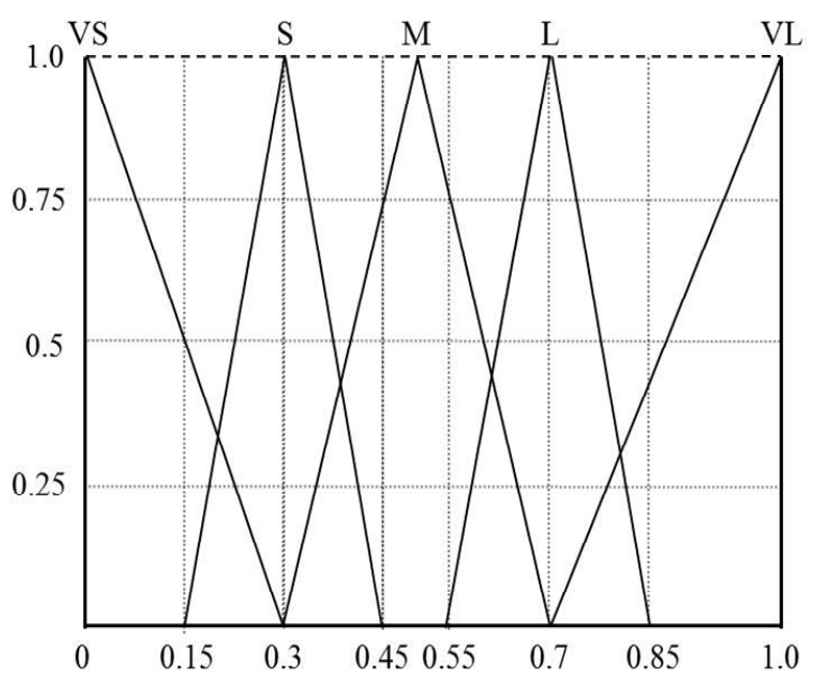

Figure 10. Fuzzy membership function for the attempts to verify an event report probability.

Table 1. Fuzzy rules.

\begin{tabular}{ccccc}
\hline \multirow{2}{*}{ Rule } & \multicolumn{2}{c}{ Input } & \multicolumn{2}{c}{ Output } \\
\cline { 2 - 5 } & $\mathrm{NN}$ & $\mathrm{NH}$ & $\mathrm{RF}$ & Probability \\
\hline 0 & Small & Small & Small & VS \\
6 & Small & Large & Small & $\mathrm{S}$ \\
10 & Medium & Small & Medium & $\mathrm{M}$ \\
17 & Medium & Large & Large & L \\
22 & Large & Medium & Medium & VL \\
\hline
\end{tabular}

"NN (neighbor nodes), NH (node's hops), RF (rate of false reports).

Table 2. Elements determining $P_{1}$ in Equation (1).

\begin{tabular}{cl}
\hline$T$ & The number of MACs in the event report \\
$N_{C}$ & The number of keys disclosed to an attacker \\
$k$ & The number of keys of a node \\
$N$ & The number of keys in the global key pool \\
\hline
\end{tabular}

The probability $P_{1}$ is used to calculate a probability that is used to filter false reports. In this paper, $P_{1}$ is a probability that is used to filter false reports. Table 3 shows the probabilities to compare the energy efficiency between the proposed method and SEF.

Equation (2) represents the probability that is used to filter false reports in SEF.

$$
P_{f s}=P_{1} \times P_{t s}\left(P_{t s}=1\right)
$$

Equation (3) represents the probability that is used to filter false reports in the proposed method.

$$
P_{f p}=P_{1} \times P_{t p}\left(0 \leq P_{t p} \leq 1\right)
$$

Table 3. Probabilities for a comparison of energy efficiency.

$P_{f s} \quad$ The probability of filtering a false report in SEF

$P_{f p} \quad$ The probability of filtering a false report in proposed method

$P_{t s} \quad$ an attempt to verify a report probability in SEF

$P_{t p} \quad$ an attempt to verify a report probability in proposed method

As shown above, $P_{t s}$ is always 1 , but $P_{t p}$ is in the range of 0 - 1 . If the verification energy consumption of a sensor node in SEF is 1, the verification energy of the node in the proposed method is always the same as or smaller than the one in SEF. We simulate this proposed method in section 4 to investigate the method.

\section{Simulation}

In this section, we explain the simulation results of the proposed method. This simulation was performed to show the energy efficiency of the proposed method compared with that of SEF. The simulation included several environments. First is the sensor field, which is $100 \mathrm{~m}$ wide and $100 \mathrm{~m}$ tall. Within this sensor field, 600 sensor nodes are deployed. A sink node in this sensor field includes 100 keys in global key pool. The global key pool is divided into 10 partitions, each of which includes 10 keys. The sensor node energies are $0.3 \mathrm{~J}$. Also, the energies that are consumed by receiving an event report are $12.5 \mu \mathrm{J}$, the energies that are consumed by sending the event report are $16.25 \mu \mathrm{J}$. Approximately 75 $\mu \mathrm{J}$ are consumed by the event report verification. The event report packet is 24 bytes. The probability that a node has a key that is not compromised $\left(P_{1}\right)$ is 0.4 . This simulation was divided into two aspects. The first aspect is energy efficiency. A simulation comparing energy efficiency was made between SEF and the proposed method. The simulation was tested in two environments: when a rate of false reports which were generated by sensor nodes in the sensor field was $10 \%$, and when the rate of false reports was $30 \%$. Figure 11 compares SEF and the proposed method in terms of energy consumption of sensor nodes when the rate of false reports was $10 \%$.

Figure 11 shows that less sensor node energy was consumed in the proposed method than in the SEF when the rate of false reports was $10 \%$. We found that 3.5\% less energy was consumed in the proposed method than that in SEF on average. Figure 12 compares SEF and the proposed method in terms of energy consumption of the sensor nodes when the rate of false reports was $30 \%$.

Figure 12 also indicates that less sensor node energy was consumed in the proposed method than in the SEF when the rate of false reports was $30 \%$. We found that $3 \%$ 


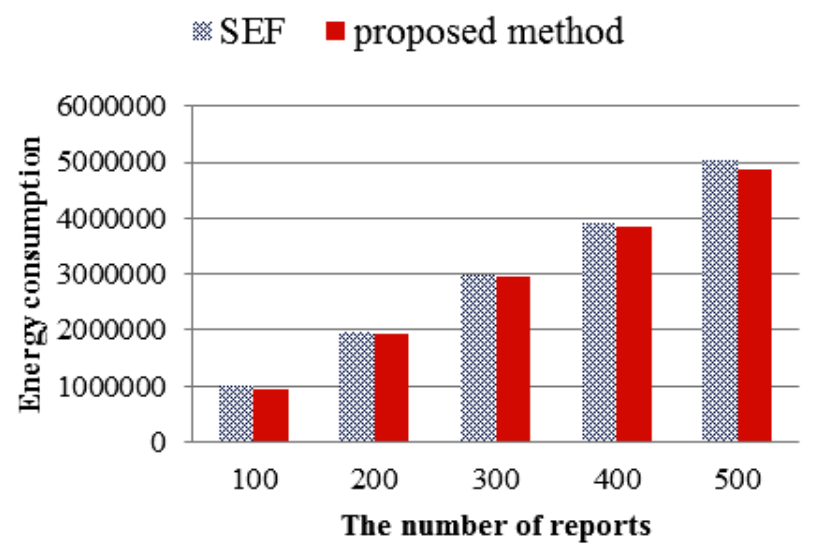

Figure 11. Comparison of energy consumption (The rate of false reports is $10 \%)$.

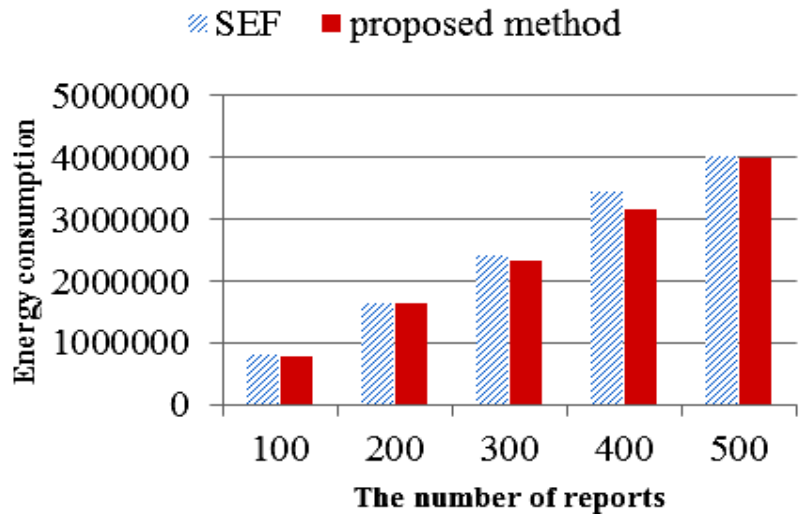

Figure 12. Comparison of energy consumption (The rate of false reports is $30 \%$ ).

less energy was consumed in the proposed method than that in SEF on average. Figures $\mathbf{1 3}$ and $\mathbf{1 4}$ indicate that when the false report rate was low in a sensor network, the energy efficiency of the proposed method was greater than that of the SEF.

The second aspect is security. Because the proposed method controls probability using intermediate nodes to verify an event report in the sensor network, the security of the proposed method has to be tested and compared against that of the SEF. Thus, this simulation also was tested in two environments. Figure $\mathbf{1 3}$ compares the number of false reports in SEF with the number of false reports that were not filtered by sensor nodes in en-route filtering in the proposed method when the false report rate was $10 \%$.

Figure 13 shows that the number of false reports that were not filtered by en-route filtering in the proposed method is similar to the number of false reports in SEF. Actually, an average of 0.03 more false reports was seen in the proposed method than the average seen in SEF.

Figure 14 compares the number of false reports in SEF with the number of false reports that were not filtered

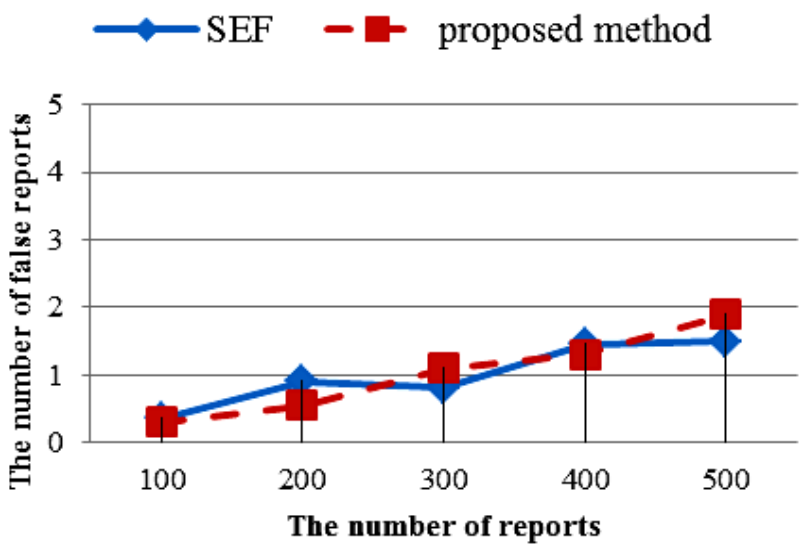

Figure 13. Comparison of the number of false reports (The rate of false reports is $10 \%$ ).

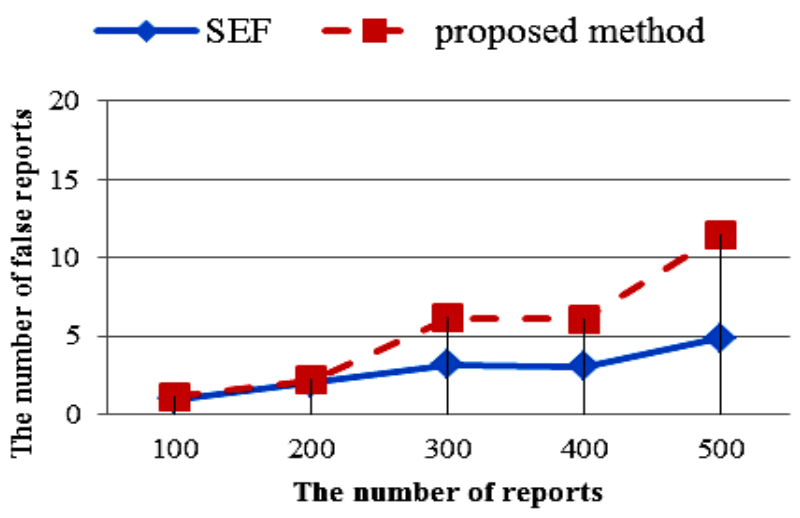

Figure 14. Comparison of the number of false reports (The rate of false reports is $30 \%$ ).

by en-route filtering in the proposed method when the false report rate was $30 \%$.

Figure 14 shows that the number of false reports that were not filtered by en-route filtering between SEF and proposed method is again similar. An average of 2.61 more false reports was seen in the proposed method than the average seen in SEF. As shown above, the security level of the proposed method is similar to that of SEF. Moreover, both SEF and the proposed method contain a sink verification step in which all unfiltered false reports are dropped. Thus, the energy efficiency of the proposed method is the more important factor.

\section{Conclusions}

Sensor networks, which are used in open environments, are vulnerable to physical attacks from the outside. A false report injection attack is a physical attack in which a node compromised by an attacker forwards many false reports that are not based on real events. Sensor node energy is thus wasted by the attack. However, there are many solutions that defend against false report injection 
attacks. One such solution is SEF, in which any time a node in the sensor network receives an event report, it verifies the validity of that report using a fixed probability. If the event report is false, the node drops it. Thus, SEF prevents energy waste by filtering false reports early. However, if the false report rate in the sensor network is low, sensor node energy is wasted because the nodes in SEF verify both false and legitimate reports as the same probability. Thus, in this paper, we suggested a method by which each sensor node controls The probability is determined by a fuzzy system. The fuzzy system of the proposed method has three inputs: the number of neighbor nodes, the number of hops from the sensor node to the sink node, and the rate of false reports among the ten most recent event reports received from a neighbor node. We performed four simulations to prove the energy efficiency of the proposed method. The first simulation compared energy consumption of SEF and the proposed method for various false reports rates. We also compared the number of false reports that were not filtered. Thus, our proposed method can be respected for its energy efficiency in sensor network.

\section{Acknowledgements}

This research was supported by Basic Science Research Program through the National Research Foundation of Korea (NRF) funded by the Ministry of Education, Science and Technology (No. 2011-0004955).

\section{References}

[1] I. F. Akyildiz, W. L. Su, Y. Sankarasubramaniam and E. Cayirci, "A Survey on Sensor Networks," IEEE Communications Magazine, Vol. 40, No. 8, 2002, pp. 102-114. doi:10.1109/MCOM.2002.1024422

[2] F. Li and J. Wu, "A Probabilistic Voting-Based Filtering Scheme in Wireless Sensor Networks," Proceedings of the 2006 International Conference on Wireless Communications and Mobile Computing, Vancouver, 3-6 July 2006, pp. 27-32.

[3] B. Przydatek, D. Song and A. Perrig, "SIA: Secure Information Aggregation in Sensor Networks," Proceedings of the 1st International Conference on Embedded Networked Sensor Systems, Los Angeles, 5-7 November 2003.

[4] F. Ye, H. Y. Luo, S. W. Lu and L. X. Zhang, "Statistical En-Route Filtering of Injected False Data in Sensor Networks," 23rd Annual Joint Conference of the IEEE Computer and Communications Societies, Vol. 4, No. 7-11, 2004, pp. 2446-2457.

[5] H. Yang and S. W. Lu, "Commutative Cipher Based En-Route Filtering in Wireless Sensor Networks," IEEE 60th Vehicular Technology Conference, Vol. 2, Los Angeles, 26-29 September 2004, pp. 1223-1227

[6] C. Karlof and D. Wagner, "Secure Routing in Wireless Sensor Networks: Attacks and Countermeasures," Proceedings of the First IEEE: 2003 IEEE International Workshop on Sensor Network Protocols and Applications, Anchorage, 11 May 2003, pp. 113-127. 